\title{
Implicaciones legales de la hemotransfusión en terapia intensiva
}

\author{
Enrique Monares Zepeda, ${ }^{\star}$ Antonio Juárez Navarro, ${ }^{\ddagger}$ Orlando Rubén Pérez Nieto, ${ }^{\S}$ Manuel Nicolás Poblano Morales\|
}

\section{RESUMEN}

Antecedentes: Las hemotransfusiones son un procedimiento común en las unidades de cuidados intensivos a nivel mundial. El personal médico implicado en la hemotransfusión habitualmente no está familiarizado con el marco legal de tal disposición. Caso clínico: Paciente femenino de 17 años de edad ingresada en la $\mathrm{UCl}$ con politraumatismo, choque hemorrágico severo, traumatismo craneoencefálico severo meritorio de craneotomía descompresiva urgente, hemoneumotórax y lesión esplénica; tales diagnósticos le generan choque hemorrágico con anemia severa por hemoglobina en $5 \mathrm{~g} / \mathrm{dL}$. La madre de la paciente, responsable de la misma, prohíbe que se realice transfusión de hemoderivados por ideas religiosas. Los médicos a cargo solicitan apoyo de expertos en el área legal para saber cuál es la mejor conducta a seguir con efecto de anticipar y prevenir los posibles conflictos legales, quienes explican que la vida no es un bien jurídico disponible y, ante condiciones de gravedad, la hemotransfusión debe realizarse.

Conclusiones: Es necesario establecer una política legalmente válida sobre los derechos y obligaciones de transfusiones sanguíneas, que deberá ser conocida por todos los trabajadores de la salud en la institución donde se implemente dicha política, así como el derecho de solicitar asesoría legal en cada caso en que se presente en este tipo de conflictos.

Palabras clave: Choque hemorrágico, hemotransfusión, anemia, marco legal.

\section{SUMMARY}

Background: Hemotransfusions are a common procedure in intensive care units worldwide. The medical personnel involved in hemotransfusion are usually not familiar with the legal framework of such a provision.

Clinical case: A 17-year-old female admitted to the ICU with multiple trauma, severe hemorrhagic shock, severe cranioencephalic traumatism, urgent decompressive craniotomy, hemopneumothorax and splenic lesion, such diagnoses generate hemorrhagic shock with severe anemia due to hemoglobin at $5 \mathrm{~g} / \mathrm{dL}$. The mother of the patient, responsible for her, prohibits any transfusion of blood products by religious idea. The doctors in charge request expert support in the legal area to know what is the best behavior to follow, with the effect of anticipating and preventing possible legal conflicts, who explain that life is not a legal right available, and in the face of serious conditions, the hemotransfusion must be done.

Conclusions: A legally valid policy should be established on the rights and obligations of blood transfusions, which should be known by all health workers in the institution where said policy is implemented and that of requesting legal advice in each case presented in this type of conflicts.

Key words: Hemorrhagic shock, blood transfusion, anemia, legal.

\section{RESUMO}

Antecedentes: A hemotransfusão é um procedimento comum em unidades de terapia intensiva em todo o mundo. O pessoal médico envolvido na hemotransfusão geralmente não está familiarizado com a estrutura legal de tal disposição.

Caso clínico: Paciente do sexo feminino, 17 anos, admitida na UTI com politraumatismo, choque hemorrágico grave, trauma cranioencefálico grave que necessitou craniotomia descompressiva de urgência, hemopneumotórax e lesão esplênica, tais diagnósticos ocasionaram choque hemorrágico com anemia grave devido à hemoglobina de $5 \mathrm{~g} / \mathrm{dL}$.

A mãe da paciente, responsável pela mesma, proíbe qualquer transfusão de hemoderivados por crença religiosa.

Os médicos responsáveis solicitam apoio especializado na área jurídica para saber qual a melhor conduta a seguir, com a intenção de antecipar e prevenir possíveis conflitos legais, que explicam que a vida não é um direito legal disponível, e diante de condições graves, a hemotransfusão deve ser feita.

Conclusões: Deve ser estabelecida uma política legalmente válida sobre os direitos e obrigações das hemotransfusões, que deve ser do conhecimento de todos os profissionais de saúde na instituição onde tal política é implementada e da solicitação de assessoria jurídica em cada caso que se apresente esse tipo de conflitos.

Palavras-chave: Choque hemorrágico, hemotransfusão, anemia, arcabouço legal.

\footnotetext{
* Hospital San Ángel Inn Universidad, CDMX.

‡ Blindaje Médico Jurídico «Antonio Juárez y Asociados», DHC.

$\S$ Hospital General «San Juan del Río», Qro.

" Hospital H+, Santiago de Querétaro.
}

Recepción: 23/03/2018. Aceptación: 10/05/2018.

Este artículo puede ser consultado en versión completa en http://www.medigraphic.com/medicinacritica

\section{ANTECEDENTES}

Las hemotransfusiones son un procedimiento común en las unidades de cuidados intensivos a nivel mundial. Las tasas de transfusión son variables y se reporta que entre 20 y $53 \%$ de los pacientes reciben sangre en forma de concentrado eritrocitario durante su estancia en la unidad de cuidados intensivos (UCI). ${ }^{1}$ En general, se reporta un promedio de cuatro unidades de hemoderivados por paciente durante su internamiento en la $\mathrm{UCl}^{2}$ El personal médico implicado en la hemotransfusión habitualmente no está familiarizado con el marco legal de tal disposición. El actuar médico se basa en el Reglamento de la Ley General de Salud, ${ }^{3}$ que tiene por objeto proveer en la esfera administrativa el cumplimiento de la prestación de servicios de atención médica. En caso de incurrirse en un delito médico, el Código Penal Federal ${ }^{4}$ será el marco jurídico que disponga las acciones consecuentes.

\section{CASO CLÍNICO}

Paciente femenino de 17 años de edad, hospitalizada en la cama 5 de terapia intensiva, quien hace cinco horas sufrió accidente automovilístico de alta energía al encontrarse viajando en el asiento del copiloto sin cinturón de seguridad, el conductor del automóvil falleció en el acto en el lugar del accidente. La paciente es ingresada en la UCI con diagnóstico de politraumatismo y choque hemorrágico severo, presentando traumatismo craneoencefálico severo meritorio de craneotomía descompresiva urgente, así como hemoneumotórax derecho, por lo que se coloca una sonda endopleural, que presenta un drenaje inicial de $800 \mathrm{~mL}$ de contenido hemático, además se diagnostica un trauma cerrado de abdomen con líquido libre, para lo cual se efectúa una laparotomía exploradora, encontrando una lesión esplénica grado IV resuelta con esplenectomía; tales diagnósticos le generan inestabilidad hemodinámica y choque hemorrágico severo con anemia severa por una cuantificación de hemoglobina en $5 \mathrm{~g} / \mathrm{dL}$. Los padres de la paciente están actualmente divorciados. Por una parte, la madre, quien se presenta como la familiar responsable de la misma, nos notifica que prohíbe terminantemente que se realice transfusión de hemoderivados por ideología religiosa; por otra parte, el padre, quien no tiene la guarda y custodia y que no profesa dicha religión, ha exigido que se realice la transfusión, de ser necesaria. Uno de los médicos propone realizar la transfusión sin informar a la madre, en tanto que otro médico refiere que él no 
transfundirá a la paciente sin el consentimiento de esta última, dado que es la familiar responsable. A esto se agrega que la madre de la paciente y su abogado se encuentran en la sala de espera, amenazando con demandar a cualquier trabajador de la salud que participe en la transfusión no autorizada de su hija.

Dadas las circunstancias previamente expuestas, los médicos a cargo deciden solicitar apoyo de los expertos en el área legal para saber cuál es la mejor conducta a seguir con efecto de anticipar y prevenir los posibles conflictos jurídicos.

Los abogados especialistas en la materia explican que, con independencia y respeto a su libre derecho de creencia, en este caso concreto, la paciente está en situación de emergencia, en la que, de no realizarse la transfusión de hemoderivados, va a morir y, siendo que la vida no es un bien jurídico del cual se pueda disponer, es decir, que nadie puede renunciar a la vida, ni otorgar a otros la decisión de suprimir su derecho a vivir (con excepción del contexto de voluntad anticipada), nuestra obligación es proceder a la hemotransfusión mientras no existan otras alternativas científicamente comprobadas y disponibles para preservar la vida.

La recomendación de los abogados es dar aviso al Ministerio Público y proceder a la transfusión, debido a que ante la controversia de derechos debe preponderarse el derecho a la vida por encima del derecho de credo o de autodeterminación.

En todos los casos semejantes, en los cuales no exista riesgo de muerte, debe buscarse otro tipo de alternativas terapéuticas y respetar en todo momento el derecho de autodeterminación de los padres de los menores y de los usuarios en general.

Los médicos involucrados plantean las siguientes preguntas a los abogados expertos:

¿Se puede ejercer una acción legal contra un médico por transfundir hemoderivados a un paciente cuando él mismo o sus familiares se oponen por ideología religiosa o de otra índole, teniendo en claro que está en riesgo inminente de perder la vida?

La respuesta es afirmativa, dado que sí puede realizarse dicha demanda, pero se aclara que no se contaría con los elementos penales para proceder en contra del médico, ya que no violenta el derecho de autonomía, sino que trata de preservar la vida por sobre todas las cosas, acto al que están obligados y los responsables legales y/o tutores no tienen la facultad de decidir sobre la vida de sus pacientes. Un consentimiento informado que autorice dañar o dejar de actuar para preservar la vida no es legalmente válido, aclarando fuera del contexto de la voluntad anticipada.

¿Algún médico ha ido a la cárcel por no transfundir a un paciente cuya vida dependiera de ello, fuera del concepto de la voluntad anticipada?
La respuesta es afirmativa, existen casos de médicos que han sido penalmente sentenciados por no transfundir al paciente que requiere hemoderivados en el contexto de preservar la vida. Al momento de esta publicación, no hay casos de médicos que hayan sido procesados ni sentenciados por transfundir pacientes en el con el fin de preservar la vida.

\section{La vida no es un bien jurídico disponible. Explicación legal}

En principio, debemos hacer mención respecto a que existen bienes jurídicos disponibles, así como bienes jurídicos no disponibles. Cuando hablamos de la disponibilidad de los bienes jurídicos, nos referimos al derecho que se tiene a decidir sobre dichos bienes y más aún a renunciar a ellos, por ejemplo, la salud es un bien jurídico disponible, puesto que las personas podemos decidir en nuestro organismo en cuanto a tomar o no un medicamento y también respecto a realizar o no algún procedimiento médico o quirúrgico.

De lo anterior se desprende que la vida no es un bien jurídico disponible, y si bien es cierto que lo antes planteado puede confundir a los médicos, puesto que la transfusión de hemoderivados es en sí un procedimiento que influye en la salud y que en algunos casos se indica con fines terapéuticos y de rehabilitación debido a la baja de los niveles de la hemoglobina, anemias primarias, entre otras causas, también es cierto que la necesidad de transfundir a un paciente que se encuentra en circunstancias semejantes, como el caso clínico aquí presentado, cruza la línea del derecho renunciable y disponible de la salud para entrar en el ámbito del derecho a la vida; esto es, dado que los niveles de hemoglobina normales en un cuerpo humano son entre 11.7 y $15.7 \mathrm{~g} / \mathrm{dL}$ en mujeres, en el caso que nos ocupa, así como en otros muchos que suceden a diario en todo el país, la paciente, además de ser menor de edad, es decir, no es jurídicamente capaz de tomar decisiones legales, se encuentra con datos de choque hipovolémico hemorrágico severo; a saber, el volumen de circulación sanguínea es insuficiente para mantener una perfusión efectiva, lo que generará daño irreversible si no es tratada pronta y adecuadamente. Dicha situación se convierte en una emergencia que seguramente terminará con la vida de la paciente.

Aunado a lo anterior, el artículo 15 del Código Penal Federal nos ilustra al respecto tal y como se transcribe: «el delito se excluye cuando: [...] III. Se actúe con el consentimiento del titular del bien jurídico afectado, siempre que se cumplan los siguientes requisitos: a) Que el bien jurídico sea disponible [...]".

Por otro lado, se hace necesario transcribir lo normado en el artículo 7 del Código Penal Federal: ${ }^{4}$ "delito es el acto u omisión que sancionan las leyes penales. En 
los delitos de resultado material también será atribuible el resultado típico producido a quien omita impedirlo, si éste tenía el deber jurídico de evitarlo. En estos casos se considerará que el resultado es consecuencia de una conducta omisiva, cuando se determine que quien omite impedirlo tenía el deber de actuar para ello, derivado de una ley, de un contrato o de su propio actuar precedente». En otras palabras, como elementos del delito se hace necesario hablar de «omisión», «omisión simple» y «comisión por omisión». La omisión se entiende como «la ausencia del movimiento corporal esperado por la ley o que no evita la producción del daño material». La omisión simple se refiere a la «inactividad ante el deber de obrar bajo lo legalmente establecido" y la comisión por omisión se refiere a la «no evitación de la producción de un resultado material delictivo cuando se tiene la obligación de evitarlo...».

Ahora bien, el artículo 9 del Reglamento de la Ley General de Salud, en materia de prestación de servicios de atención médica, nos ilustra al respecto de forma textual: «la atención médica deberá llevarse a efecto de conformidad con los principios científicos y éticos que orientan la práctica médica», entre dichos principios éticos se encuentra el de «no maleficencia» y el de «beneficencia», motivo por el cual, si bien es cierto que el médico tiene una obligación de medios y no de resultados, también es cierto que tiene la obligación de buscar preservar la vida, así como tratar de restituir la salud y, en sí, de buscar el bien mayor para el paciente; es decir, de cometer acciones y actos tendientes a ayudar al paciente. Por ende, tiene la obligación de abstenerse de realizar omisiones que puedan dañar o más aún, ayudar a que una persona pierda la vida.

Lo anterior no tiene lugar a duda, sobre todo tratándose de menores de edad, dado que ellos no tienen la capacidad jurídica de decidir; esto es, aunque la vida llegase a ser un bien jurídico del cual se pudiera disponer, ellos no podrían disponer de dicho bien toda vez que no son sujetos de derecho de tomar esa decisión y, suponiendo sin conceder que así fuese, efectivamente sería el tutor o representante legal debidamente acreditado quien podría hacerlo. Sin embargo, en principio y partiendo de la base de que la vida no es un bien jurídico disponible, en este caso concreto, la paciente se halla en un estado de inconsciencia $y$, aunque nos encontramos ante una controversia de derecho muy común, en la que se ponen en contrapeso el derecho de autodeterminación o de libre elección, de consciencia y el derecho a la vida, es claro que siempre deberá preponderarse el bien jurídico de mayor valor, tal y como lo es el derecho a la vida.

\section{CONCLUSIONES}

Con respecto a las personas que buscan renunciar al derecho de un tercero que en ese momento se halla imposibilitado para decidir, sea o no mayor de edad, debido a su estado de gravedad y a que cuenta con un "consentimiento informado" que pretende excluir de responsabilidad al personal a cargo del paciente, se hace necesario recalcar que, en principio, ellos no son titulares de ese bien jurídico y, además, que no se trata de un bien jurídico del que se pueda disponer y, aun si lo fueran, el consentimiento sólo será eficaz en los casos en que el bien pertenezca al sujeto, que sea dado libremente y que los bienes jurídicos sean individuales. El único consentimiento que no es válido es el de la propia muerte, excepto en los casos de suicidio, dado que, por las circunstancias en las que se comete, no se puede castigar. Tampoco el consentimiento de daño al propio cuerpo es aceptable, pues la lesión en contra de las buenas costumbres está limitada por el interés de la comunidad. $Y$ de manera adicional y muy relevante, el delito de homicidio, ya sea con dolo (con la intención de llevarlo a cabo) o con culpa (cuando se deriva de una omisión de una conducta obligatoria), como en este caso es la de no dañar y la de buscar lo mejor para el paciente en cuanto al derecho a la vida, es un delito que se persigue por oficio. Por tanto, aun obteniendo las firmas de toda la familia y comunidad cercana al paciente, basta el hecho de que el Ministerio Público, quien tiene por objeto perseguir el delito, se entere para que inicie una carpeta de investigación por delito de homicidio. Pensar que esto no debe ser así sería tanto como afirmar que, al encontrar a una persona muerta con una herida por proyectil de arma de fuego en el cráneo con un documento en el que consintiera el hecho de que le dispararan y en el cual se exhibiera su firma autógrafa, debería extinguir la acción penal y evitar los mecanismos del Estado para perseguir el delito y no buscar al sujeto activo que disparó el arma; o bien a una persona que murió al ser arrollada por un vehículo automotor y que contara con un manuscrito en el que consintiera que la atropellaran o que se liberara de responsabilidad al conductor, así se hiciera y más aún, permitir que un niño renuncie a su derecho de vivir mientras se encuentra inconsciente para decidirlo y lo haga por medio de su padre o madre, que son sus responsables legales.

Por último, resaltamos que existe una ley de voluntad anticipada, la cual permite que pacientes diagnosticados de enfermedades terminales con una expectativa de vida mayor de seis meses y mediante su consentimiento ante un notario acepten «previamente» que no se les reanime en caso de caer en arresto cardiorrespiratorio y que no se les de maniobras de reanimación cardiopulmonar ni se haga nada para ayudarles. Sin embargo, independientemente de esto, dichos casos deben someterse a comités de bioética a efecto de determinar la procedencia de dicho consentimiento y las circunstancias clínicas. 
Conducta a seguir en este caso:

1) El caso debe documentarse de tal forma que no quede la menor duda de que se trata de una situación donde la única opción real y científica de preservar la vida del paciente sea la transfusión sanguínea y que no se está en el contexto de voluntad anticipada; es decir, que no tenga diagnóstico de enfermedad terminal, con una esperanza de vida menor de seis meses y con consentimiento debidamente notariado, si fuera el caso.

2) Se debe informar a la familia o responsables legales que se realizará el procedimiento $y$, en caso de que se sospeche o se tenga la certeza de que se presentará un conflicto legal, notificar inmediatamente al Ministerio Público sobre el caso, ya que de esta forma se asegura la preservación de los derechos del paciente.

Estas recomendaciones no garantizan que no se presentarán conflictos legales para el médico que proceda de esta manera, pero sí garantiza, en primer lugar, la mejor oportunidad de preservar la vida del paciente y, en segundo lugar, la mejor forma de proteger la integridad del médico ante la ley.

Se hace énfasis en que el consentimiento informado que otorga privilegios para dañar o atentar contra la vida no tiene ninguna validez legal. Cuando existe controversia entre el derecho de autonomía en las decisiones de la salud y la obligación de preservar la vida fuera de contextos de voluntad anticipada, siempre deberá preponderarse preservar la vida.

Es necesario establecer una política legalmente válida sobre los derechos y obligaciones de transfusiones sanguíneas que deberá ser conocida por todos los trabajadores de la salud en la institución donde se implemente dicha política, así como el derecho a solicitar asesoría legal en cada caso que se presente en este tipo de conflictos.

El presente artículo se realizó con la opinión de expertos, de acuerdo con las leyes vigentes y con el caso que se plantea. Cada caso deberá ser siempre revisado por expertos para su mejor solución.

\section{Agradecimiento}

Blindaje Médico Jurídico, Antonio Juárez y Asociados.

\section{BIBLIOGRAFÍA}

1. Vincent $\mathrm{JL}$, Piagnerelli M. Transfusion in the intensive care unit. Crit Care Med. 2006;34(Suppl. 5):S96-101.

2. Taylor RW, Manganaro L, O'Brien J, Trottier SJ, Parkar $\mathrm{N}$, Veremakis C. Impact of allogenic packed red blood cell transfusion on nosocomial infections rates in the critically ill patient. Crit Care Med. 2002;30(10):2249-2254.

3. Reglamento de la Ley General de Salud en Materia de Prestación de Servicios de Atención Médica. Disponible en: http://www.salud. gob.mx/unidades/cdi/nom/compi/rlgsmpsam.html

4. Código Penal Federal. Disponible en: http://www.diputados.gob. mx/LeyesBiblio/ref/cpf.htm

Correspondencia:

Enrique Monares Zepeda

Av. Río Churubusco Núm. 601,

Col. Xoco,

Del. Benito Juárez, 03339, CDMX.

Tel: (55) 56236363

Celular: 5549946531

E-mail: enrique_monares@hotmail.com, enrique_monares@hsai.com.mx 